\title{
Canonical Form Associated with an $r$-Jacobi Algebra
}

\section{Deva Michelle Bouaboté Ntoumba}

Faculty of Science and Technology, Marien NGOUABI University, Brazzaville, Congo

Email: bouabotedevamichelle@gmail.com

Received 13 November 2015; accepted 7 March 2016; published 10 March 2016

Copyright (C) 2016 by author and Scientific Research Publishing Inc.

This work is licensed under the Creative Commons Attribution International License (CC BY).

http://creativecommons.org/licenses/by/4.0/

(c) (i) Open Access

\section{Abstract}

In this paper, we denote by $A$ a commutative and unitary algebra over a commutative field $K$ of characteristic 0 and $r$ an integer $\geq 1$. We define the notion of $r$-Jacobi algebra $A$ and we construct the canonical form associated with the $r$-Jacobi algebra $A$.

\section{Keywords}

\section{Module of Kähler Differential, Lie Algebra of Order $r$, Jacobi Algebra of Order $r$}

\section{Introduction}

The concept of $n$-Lie algebra over a field $K, n$ an integer $\geq 2$, introduced by Fillipov [1], is a generalization of the concept of Lie algebra over a field $K$, which corresponds to the case where $n=2$. A structure of $n$-Lie algebra over a $K$-vector space $W$, is the given of an alternating multilinear mapping of degree $n$

$$
\{, \cdots,\}: W^{n}=W \times \cdots \times W \rightarrow W,\left(x_{1}, x_{2}, \cdots, x_{n}\right) \mapsto\left\{x_{1}, x_{2}, \cdots, x_{n}\right\}
$$

verifying the identity

$$
\left\{x_{1}, x_{2}, \cdots, x_{n-1},\left\{y_{1}, y_{2}, \cdots, y_{n}\right\}\right\}=\sum_{i=1}^{n}\left\{y_{1}, y_{2}, \cdots, y_{i-1},\left\{x_{1}, x_{2}, \cdots, x_{n-1}, y_{i}\right\}, y_{i+1}, \cdots, y_{n}\right\}
$$

for all $x_{1}, x_{2}, \cdots, x_{n-1}, y_{1}, y_{2}, \cdots, y_{n} \in W$. This identity is called Jacobi identity of $n$-Lie algebra $W$ [1] [2].

A derivation of an $n$-Lie algebra $(W,\{, \cdots\}$,$) is a K$-linear map

$$
D: W \rightarrow W
$$

such that 


$$
D\left\{x_{1}, x_{2}, \cdots, x_{n}\right\}=\sum_{i=1}^{n}\left\{x_{1}, \cdots, D\left(x_{i}\right), \cdots, x_{n}\right\}
$$

for all $x_{1}, x_{2}, \cdots, x_{n} \in W$.

The set of all derivations of a $n$-Lie algebra $W$ is a $K$-Lie algebra denoted by $\operatorname{Der}_{K}(W)$.

If $(W,\{, \cdots\}$,$) is a n$-Lie algebra, then for all $x_{1}, x_{2}, \cdots, x_{n-1} \in W$, the map

$$
\operatorname{ad}\left(x_{1}, x_{2}, \cdots, x_{n-1}\right): W \rightarrow W, y \mapsto\left\{x_{1}, x_{2}, \cdots, x_{n-1}, y\right\}
$$

is a derivation of $(W,\{, \cdots\}$,$) .$

When $A$ is a commutative algebra, with unit $1_{A}$ over a commutative field $K$ of characteristic zero, and when $M$ is a $A$-module, a linear map

$$
\partial: A \rightarrow M
$$

is a differential operator of order $\leq 1$ [3] [4] if, for all $a$ and $b$ belonging to $A$,

$$
\partial(a b)=\partial(a) \cdot b+a \cdot \partial(b)-a b \cdot \partial\left(1_{A}\right) .
$$

When $\partial\left(1_{A}\right)=0$, we have the usual notion of derivation from $A$ into $M$.

We denote by $\operatorname{Diff}_{K}(A, M)$ the $A$-module of differential operator of order $\leq 1$ from $A$ into $M$ and by $\operatorname{Diff}_{K}(A)$ the $A$-module of differential operator of order $\leq 1$ on $A(M=A)$.

The aim of this work is to define the notion of $r$-Jacobi algebra and to construct the canonical form associated with this $r$-Jacobi algebra.

In the following, $A$ denotes a unitary commutative algebra over a commutative field $K$ of characteristic zero with unit $1_{A}$ and $\Omega_{K}(A)$ the module of Kähler differential of $A$ and

$$
d_{A / K}: A \rightarrow \Omega_{K}(A), a \mapsto d_{A / K}(a)
$$

the canonical derivation [3] [4].

\section{Structure of Jacobi Algebra of Order $r \geq 1$}

\section{$A$-Module $A \times \Omega_{K}(A)$}

Proposition 1 [3] The map $D_{A / K}: A \rightarrow A \times \Omega_{K}(A), a \mapsto\left(a, d_{A / K}(a)\right)$ is a differential operator of order $\leq 1$. Moreover the image of $D_{A / K}$ generates the A-module $A \times \Omega_{K}(A)$.

The pair $\left(A \times \Omega_{K}(A), D_{A / K}\right)$ has the following universal property [3] [5] [6]: for all $A$-module $M$ and for all differential operator of order $\leq 1$

$$
\varphi: A \rightarrow M
$$

there exists an unique $A$-linear map

$$
\tilde{\varphi}: A \times \Omega_{K}(A) \rightarrow M
$$

such that

$$
\tilde{\varphi} \circ D_{A / K}=\varphi .
$$

Moreover, the map

$$
\operatorname{Hom}_{A}\left(A \times \Omega_{K}(A), M\right) \rightarrow \operatorname{Diff}_{K}(A, M), \psi \mapsto \psi \circ D_{A / K}
$$

is an isomorphism of $A$-modules.

For all integer $p \geq 1$, we say that an alternating $K$-multilinear map

$$
\varphi: A^{p}=A \times A \times \cdots \times A \rightarrow M
$$

is a alternating $p$-differential operator if for all $a_{1}, a_{2}, \cdots, a_{p} \in A$, the map 


$$
\varphi^{i}: A \rightarrow M, a_{i} \mapsto \varphi\left(a_{1}, a_{2}, \cdots, a_{i-1}, a_{i}, a_{i+1}, \cdots, a_{p}\right)
$$

is a alternating differential operator of order $\leq 1$ for all $i=1,2, \cdots, p$.

We denote by $\mathcal{L}_{\text {alt }}^{p}\left(A \times \Omega_{K}(A), M\right)$, the $A$-module of alternating $A$-multilinear maps of degree $p$ from $A \times \Omega_{K}(A)$ into $M$ and $\operatorname{Diff}_{\text {alt }}^{p}(A, M)$, the $A$-module of alternating $p$-differential operators from $A$ into $M$.

One notes

$$
D_{A / K}^{(p)}=D_{A / K} \times D_{A / K} \times \cdots \times D_{A / K}: A^{p} \rightarrow\left[A \times \Omega_{K}(A)\right]^{p}
$$

such that

$$
D_{A / K}^{(p)}\left(a_{1}, a_{2}, \cdots, a_{p}\right)=\left(D_{A / K}\left(a_{1}\right), D_{A / K}\left(a_{2}\right), \cdots, D_{A / K}\left(a_{p}\right)\right)
$$

for all $a_{1}, a_{2}, \cdots, a_{p} \in A$.

When $\Lambda_{A}\left[A \times \Omega_{K}(A)\right]=\bigoplus_{n \in \mathbb{N}} \Lambda_{A}^{n}\left[A \times \Omega_{K}(A)\right]$ is the $A$-exterior algebra of the $A$-module $A \times \Omega_{K}(A)$, the differential operator

$$
D_{\mathrm{A} / K}: A \rightarrow A \times \Omega_{K}(A)
$$

can be extended into a differential operator again noted

$$
D_{A / K}: \Lambda_{A}\left[A \times \Omega_{K}(A)\right] \rightarrow \Lambda_{A}\left[A \times \Omega_{K}(A)\right]
$$

of degree +1 and of square 0 . Thus, the pair $\left(\Lambda_{A}\left[A \times \Omega_{K}(A)\right], D_{A / K}\right)$ is a differential complex [3].

For all $A$-module $M$ and for all alternating $p$-differential operator

$$
\varphi: A^{p} \rightarrow M,
$$

there exists an unique alternating $A$-multilinear map of degree $p$

$$
\tilde{\varphi}:\left[A \times \Omega_{K}(A)\right]^{p} \rightarrow M
$$

such that

$$
\tilde{\varphi} \circ D_{A / K}^{(p)}=\varphi .
$$

Thus, the existence of an unique $A$-linear map

$$
\bar{\varphi}: \Lambda_{A}^{p}\left[A \times \Omega_{K}(A)\right] \rightarrow M
$$

such that

$$
\bar{\varphi}\left(D_{A / K}\left(a_{1}\right) \Lambda D_{A / K}\left(a_{2}\right) \Lambda \cdots \Lambda D_{A / K}\left(a_{p}\right)\right)=\varphi\left(a_{1}, a_{2}, \cdots, a_{p}\right)
$$

for all $a_{1}, a_{2}, \cdots, a_{p}$ elements of $A$ when the map

$$
\varphi: A^{p} \rightarrow M,
$$

is a alternating $p$-differential operator. Moreover, the map

$$
\mathcal{L}_{\text {alt }}^{p}\left(A \times \Omega_{K}(A), M\right) \rightarrow \operatorname{Diff}_{\text {alt }}^{p}(A, M), f \mapsto f \circ D_{A / K}^{(p)}
$$

is an isomorphism of $A$-modules [3].

\section{Structure of $r$-Jacobi Algebra}

We say that a commutative algebra with unit $A$ on a commutative field $K$ of characteristic zero, is a $r$-Jacobi algebra, $r \geq 1$ an integer, if $A$ is provided with a structure of $2 r$-Lie algebra over $K$ of bracket $\{, \cdots$,$\} , such$ 
that for all $\left(a_{1}, a_{2}, \cdots, a_{2 r-1}\right) \in A^{2 r-1}$, the map

$$
\operatorname{ad}\left(a_{1}, a_{2}, \cdots, a_{2 r-1}\right): A \rightarrow A, b \mapsto\left\{a_{1}, a_{2}, \cdots, a_{2 r-1}, b\right\}
$$

is a differential operator of order $\leq 1$.

Proposition 2 When A is a r-Jacobi algebra, then there exist an unique A-linear map

$$
\overline{a d}: \Lambda_{A}^{2 r-1}\left[A \times \Omega_{K}(A)\right] \rightarrow \operatorname{Diff}_{K}(A)
$$

such that, for all $\left(a_{1}, a_{2}, \cdots, a_{2 r-1}\right) \in A$

$$
\overline{a d}\left(D_{A / K}\left(a_{1}\right) \Lambda D_{A / K}\left(a_{2}\right) \Lambda \cdots \Lambda D_{A / K}\left(a_{2 r-1}\right)\right)=\operatorname{ad}\left(a_{1}, a_{2}, \cdots, a_{2 r-1}\right) .
$$

Proof. The map

$$
\text { ad }: A^{2 r-1} \rightarrow \operatorname{Diff}_{K}(A),\left(a_{1}, a_{2}, \cdots, a_{2 r-1}\right) \mapsto a d\left(a_{1}, a_{2}, \cdots, a_{2 r-1}\right)
$$

is an alternating $(2 r-1)$-differential operator. Thus deduced the existence and the uniqueness of the $A$-linear map

$$
\overline{a d}: \Lambda_{A}^{2 r-1}\left[A \times \Omega_{K}(A)\right] \rightarrow \operatorname{Diff}_{K}(A)
$$

such that

$$
\overline{a d}\left(D_{A / K}\left(a_{1}\right) \Lambda D_{A / K}\left(a_{2}\right) \Lambda \cdots \Lambda D_{A / K}\left(a_{2 r-1}\right)\right)=\operatorname{ad}\left(a_{1}, a_{2}, \cdots, a_{2 r-1}\right) .
$$

That ends the proof.

\section{Canonical form Associated with a $r$-Jacobi Algebra}

In what follows, $A$ is a $r$-Jacobi algebra.

Theorem 3 The map

$$
A^{2 r} \rightarrow A,\left(a_{1}, a_{2}, \cdots, a_{2 r-1}, a_{2 r}\right) \mapsto(1-2 r) \cdot\left\{a_{1}, a_{2}, \cdots, a_{2 r-1}, a_{2 r}\right\}
$$

is an alternating $2 r$-differential operator and induces an alternating A-multilinear mapping and only one of degree $2 r$

$$
\omega_{2 r}:\left[A \times \Omega_{K}(A)\right]^{2 r} \rightarrow A
$$

such that

$$
\omega_{2 r}\left(D_{A / K}\left(a_{1}\right), \cdots, D_{A / K}\left(a_{2 r-1}\right), D_{A / K}\left(a_{2 r}\right)\right)=(1-2 r) \cdot\left\{a_{1}, a_{2}, \cdots, a_{2 r-1}, a_{2 r}\right\} .
$$

Proof. As the map

$$
\operatorname{ad}\left(a_{1}, a_{2}, \cdots, a_{2 r-1}\right): A \rightarrow A, b \mapsto\left\{a_{1}, a_{2}, \cdots, a_{2 r-1}, b\right\}
$$

is a $A$-differential operator of order $\leq 1$ and the map

$$
a d: A^{2 r-1} \rightarrow \operatorname{Diff}_{K}(A),\left(a_{1}, a_{2}, \cdots, a_{2 r-1}\right) \mapsto \operatorname{ad}\left(a_{1}, a_{2}, \cdots, a_{2 r-1}\right)
$$

is an alternating $(2 r-1)$-differential operator.

The unique $A$-alternating multinear map of degree $2 r$

$$
\omega_{2 r}:\left[A \times \Omega_{K}(A)\right]^{2 r} \rightarrow A
$$

induce an unique $A$-linear map

$$
\omega: \Lambda_{A}^{2 r}\left[A \times \Omega_{K}(A)\right] \rightarrow A
$$


such that

$$
\omega\left(D_{A / K}\left(a_{1}\right) \Lambda \cdots \Lambda D_{A / K}\left(a_{2 r-1}\right) \Lambda D_{A / K}\left(a_{2 r}\right)\right)=(1-2 r) \cdot\left\{a_{1}, a_{2}, \cdots, a_{2 r-1}\right\}
$$

for all $a_{1}, a_{2}, \cdots, a_{2 r} \in A$.

We say that $\omega$ is the canonical form associated with the $r$-Jacobi algebra $A$.

Corollary 1 For all $u \in \Lambda_{A}^{2 r-1}\left[A \times \Omega_{K}(A)\right]$,

$$
[\overline{a d}(u)](a)=(1-2 r)^{-1} \cdot \omega\left(u \Lambda D_{A / K}(a)\right)
$$

for any $a \in A$.

\section{Acknowledgements}

The author thanks Prof. E. Okassa for his remarks and sugestions.

\section{References}

[1] Fillipov, V.T. (1985) N-Lie Algebra. Sib. Mat. J., 26, 126-140.

[2] Bossoto, B.G.R., Okassa, E. Omporo, M. Lie algebra of an n-Lie algebra. Arxiv: 1506.06306v1.

[3] Okassa, E. (2007) Algèbres de Jacobi et algèbres de Lie-Rinehart-Jacobi. Journal of Pure and Applied Algebra, 208, 1071-1089. http://dx.doi.org/10.1016/j.jpaa.2006.05.013

[4] Okassa, E. (2008) On Lie-Rinehart-Jacobi Algebras. Journal of Algebras and Its Aplications, 7, 749-772.

[5] Bourbaki, N. (1970) Algèbre. Chapitres 1 à 3. Hermann, Paris.

[6] Bourbaki, N. (1980) Algèbre. Chapitre 10, Algèbre Homologique. Masson, Paris, New York, Barcelone, Milan. 